\title{
SUPERGIANT STARS AS TRACERS OF \\ GALACTIC CHEMICAL COMPOSITION
}

\author{
A ARELLANO FERRO AND L PARRAO \\ Instituto de Astronomia, UNAM, Mexico
}

AND

L MANTEGAZZA

Dip. di Fisica Nucleare e Teorica, Universita di Pavia, Italia

A photometric reddening-free calibration for $[\mathrm{Fe} / \mathrm{H}]$ valid for giant and supergiant stars of intermediate temperature, has been obtained using the Strömgren $u v b y \beta$ system. Galactic supergiants, supergiants in Magellanic Clouds and galactic metal deficient red giants with spectroscopic determinations of $[\mathrm{Fe} / \mathrm{H}]$ were used as calibrators. The calibration can be used to predict $[\mathrm{Fe} / \mathrm{H}]$ with an accuracy of 0.33 dex and shows the potential of supergiant stars as tracers of iron abundances in other galaxies. 